\section{THE}

CARTER CENTER

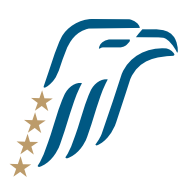

\title{
Mental Health
}

Program

Waging Peace. Fighting Disease. Building Hope.

\section{What is a mental illness?}

Mental illnesses are health conditions characterized by impairment of an individual's cognitive, emotional, or behavioral functioning, and caused by social, psychological, biochemical, genetic, or other factors. Mental illnesses are among the most common health conditions in the United States: one in four Americans will experience a diagnosable mental illness in a given year.

Although even the most serious mental illnesses can be effectively treated, the greatest barrier for Americans accessing mental health care is the stigma and resulting discrimination associated with these illnesses. In fact, 60 percent of people with mental illnesses do not receive treatment (Kessler, 2006).

\section{What is Mrs. Carter's} \section{involvement in mental health issues?}

Former First Lady Rosalynn Carter is known worldwide as a leader and proponent in the field of mental health.

Mrs. Carter has worked tirelessly for more than 40 years to improve the quality of life for those living with mental illnesses and their families, and to advance mental health services. Her work began when President Carter was Governor of Georgia and continued during the White House years with a president's commission on mental health. Her current mental health work continues through the Carter Center Mental Health Program, where projects include informing public policy and reducing stigma and discrimination against mental illnesses.
In partnership with her husband, former U.S. President Jimmy Carter, Mrs. Carter co-founded The Carter Center in 1982 and the Center's Mental Health Program in 1991.

\section{What are the goals of the Carter Center's Mental Health Program?}

The Carter Center's Mental Health Program is known for acting as an honest broker between advocates, policymakers, and consumers of mental health services to support reform and improvement in the field. The program's four strategic goals are:

- To reduce stigma and discrimination against people with mental illnesses
- To achieve equity for mental health care comparable to other health care

- To advance promotion, prevention, and early intervention services for children and their families

- To increase public awareness worldwide about mental health and mental illness and to stimulate local actions to address those issues

\section{What are some of the activities of the Mental Health Program?}

Mrs. Carter chairs the Center's Mental Health Task Force - an advisory body of experts, advocates, and consumers of mental health services that promotes positive change in the field.

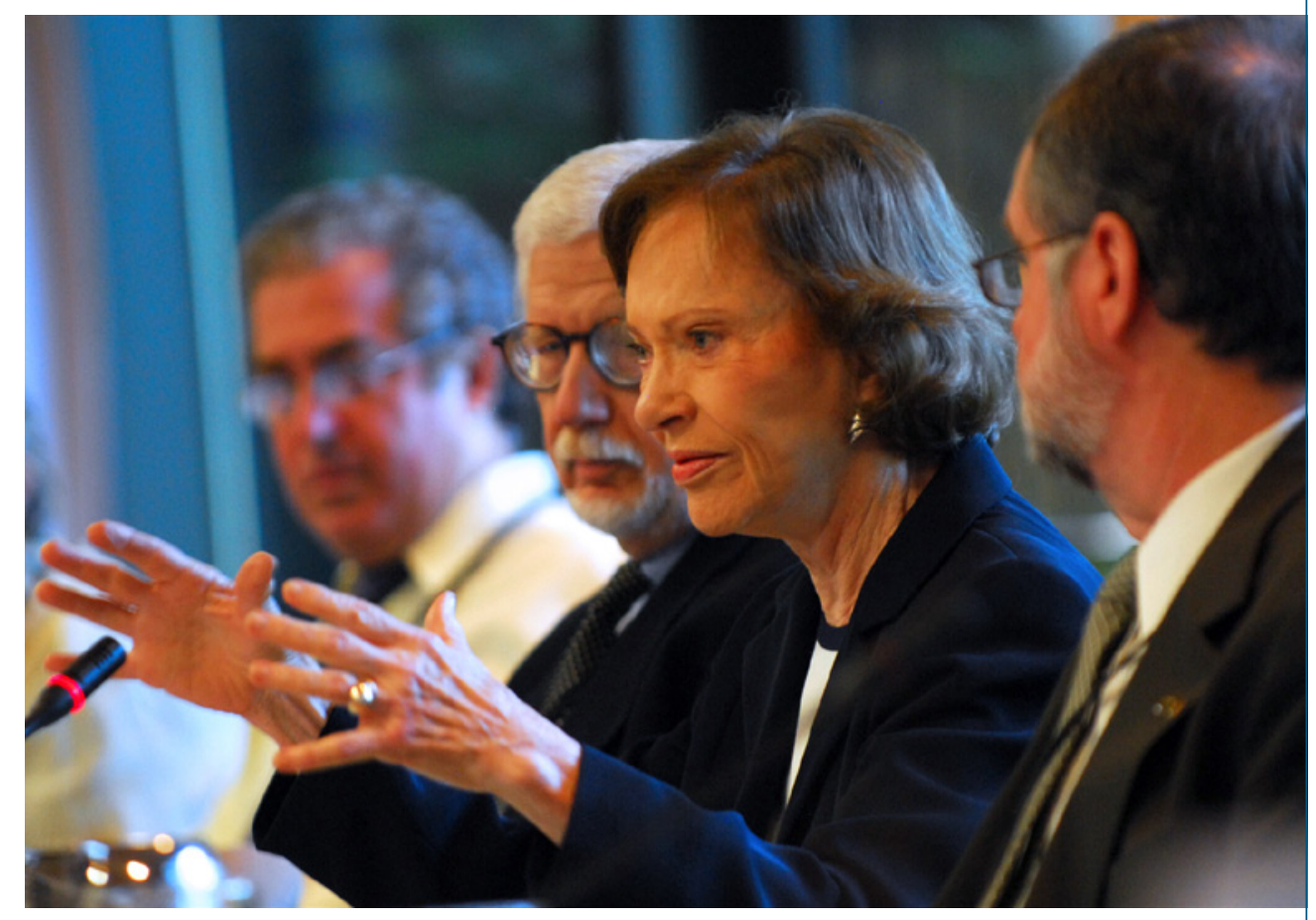




\section{Mental Health Program}

The Carter Center hosts two publicpolicy forums each year for those in the mental health field: the Rosalynn Carter Georgia Mental Health Forum, which focuses on issues within the state of Georgia, and the Rosalynn Carter Symposium on Mental Health Policy, which addresses major challenges in the mental health field.

Because journalists in all forms of media play an increasingly important role in shaping public understanding and debate about health care issues, the Rosalynn Carter Fellowships for Mental Health Journalism were established to help journalists accurately report on a wide range of mental health issues.

Projects resulting from the Rosalynn Carter Fellowships for Mental Health Journalism have the potential to greatly reduce stigma and better inform people about pressing mental health issues. Since the fellowships were established in 1996, fellows have produced more than 1,400 stories, documentaries, books, and other works during and after their fellowship year. Their projects have garnered an Emmy award, nominations for the Pulitzer Prize, and awards from Mental Health America, the American Psychological Association, Amnesty International, and the Association of Health Care Journalists.

In 2008, the Mental Health Program launched a multi-year project, the Primary Care Initiative, which works to improve access to mental health care in primary care settings.

The initiative brings together various stakeholders to identify policy barriers to real-world implementation and to develop and monitor action steps.

Building upon nearly two decades of Carter Center efforts to foster peace and democracy in Liberia, the Carter Center Mental Health Program in 2010 launched a five-year initiative to help create a sustainable mental health sys- tem in Liberia that will address a broad range of mental health conditions. The overarching goal of the Carter Center's Liberia Mental Health Program initiative is to improve functioning in people with mental illnesses in the most populous counties of Liberia.

The initiative will assist the Liberia Ministry of Health and Social Welfare in the following ways: building local training for mental health professionals, such as nurses; collaborating on implementation of the national mental health plan; developing support models for family caregivers; promoting advocacy; and working to reduce stigma and discrimination against people with mental illnesses.

Finally, since 2008, The Carter Center has been working with local mental health stakeholders to bring forward solutions to help the government address Georgia's crumbling public mental health care systems. 